SLAC-PUB-9928

June 2003

\title{
Calculation and Optimization of Laser Acceleration in Vacuum*
}

\author{
Z. Huang, G. Stupakov \\ Stanford Linear Accelerator Center, Stanford University, Stanford, CA 94309 \\ M. Zolotorev \\ Center for Beam Physics, Lawrence Berkeley National Laboratory, Berkeley, CA 94720
}

\begin{abstract}
Extraordinarily high fields generated by focused lasers are envisioned to accelerate particles to high energies. In this paper, we develop a new method to calculate laser acceleration in vacuum based on the energy exchange arose from the interference of the laser field with the radiation field of the particle. We apply this method to a simple accelerating structure, a perfectly conducting screen with a round hole, and show that how to optimize the energy gain with respect to the hole radius, laser angle and spot size, as well as the transverse profile of the laser. Limitation and energy scaling of this acceleration method are also discussed.
\end{abstract}

Submitted to Phys. Rev. ST Accel. Beams

*Work supported by Department of Energy contracts DE-AC03-76SF00515 and DE-AC03-78SF00098. 


\section{INTRODUCTION}

Acceleration of charged particles by laser fields in vacuum can be calculated as

$$
\Delta U_{\mathrm{acc}}=e \int \mathbf{E} \cdot \mathbf{v} d s
$$

where $\Delta U_{\text {acc }}$ is the energy gain, $e$ is the charge, $\mathbf{E}$ is the electric field, $\mathbf{v}$ is the particle's velocity, and the integral is taken along the particle's path. In a straight orbit approximation, when $\mathbf{v}$ in Eq. (1) is considered as a constant unperturbed velocity, according to Lawson-

Woodward theorem [1], laser acceleration in vacuum is only possible in a close proximity to material boundaries. The acceleration occurs because currents and charges induced by the laser field in the material distort the incident electromagnetic field in a way which gives a nonzero value for the integral.

A direct calculation of the integral in Eq. (1) requires solving Maxwell's equations in the vicinity of the material boundaries. In most cases, this leads to a formidable electromagnetic problem and requires extensive numerical computations. Only very simple geometries of the problem allow an analytical calculation of the energy gain directly from Eq. (1) (see, e.g., $[2,3])$.

In this paper we develop a new method to calculate the energy gain $\Delta U_{\text {acc }}$. It is based on the energy balance equation for the electromagnetic field energy and the particle's energy, and requires only knowledge of the radiation field in the far zone. In its most general formulation, it is no limited to vacuum and straight orbits - it can also be used for acceleration in a medium (e.g., inverse Cherenkov acceleration), and curvilinear orbits (such as in inverse FEL acceleration). Palmer mentions this method in Ref. [1] in the context of one of the explanations of the mechanisms of acceleration.

To demonstrate advantages of the new method, we apply it to a relatively simple problem: laser acceleration of a particle passing through a round hole in a perfectly conducting metal screen. The assumption of perfect conductivity of the metal is valid if the laser frequency is smaller than the plasma frequency for the metal. Two different laser illuminations are considered: first with a higher-order laser mode, and second with two-crossed Gaussian laser beams. Note that crossed Gaussian lasers are used in the LEAP experiment at Stanford University [4] and in the proposed E-163 experiment at SLAC [5]. In the limit when the hole radius $a$ tends to zero, we show that our result agrees with direct calculation of the 
integral (1). Taking into account the effect of a damage threshold for materials, we show how our calculations allow also to optimize the gain for given laser parameters, and find the limits of this acceleration method.

\section{RELATION BETWEEN RADIATION FIELDS AND ENERGY GAIN}

Consider a bunch passing through a hole in a perfectly conducting metal screen, as shown in Fig. 1. The hole may have arbitrary shape, although in subsequent sections we will assume that it is round, with radius $a$. At the time of passage, the bunch is irradiated by a laser pulse, and due to the interaction with the laser light, particles in the bunch will be accelerated or decelerated depending on the phase of the laser wave.

We introduce a surface of large radius $R$ enclosing a volume $V$ which includes the acceleration area. Eventually, we will assume $R \rightarrow \infty$. Initially, at $t \rightarrow-\infty$, a particle in the bunch and a laser pulse are located outside of the surface $S$. After the interaction, when $t \rightarrow \infty$, they will leave the volume $V$.

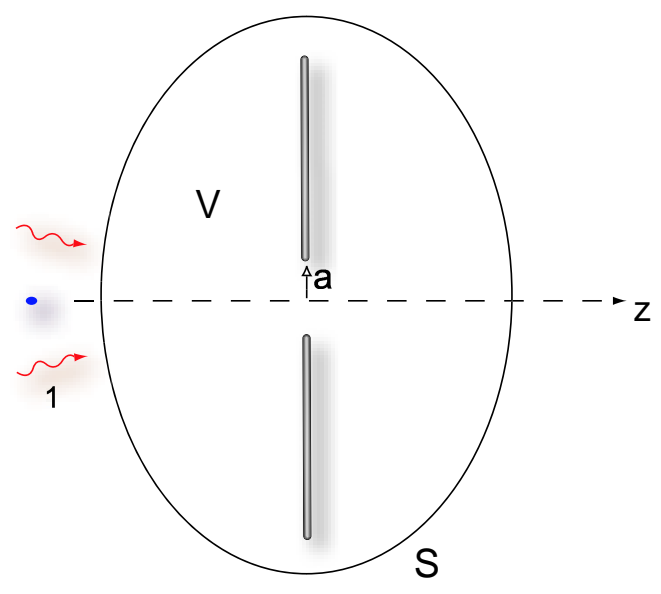

FIG. 1: Layout of a vacuum laser acceleration experiment. A perfectly conducting screen with a round hole of radius $a$ is located at $z=0$. Initially, a particle and a laser pulse are at position 1 outside of the volume $V$.

We will use the energy balance equation for the electromagnetic field (see, e. g. [6, 7]):

$$
\frac{\partial}{\partial t} \int d V \frac{E^{2}+H^{2}}{8 \pi}+\int_{V} d V \mathbf{j} \cdot \mathbf{E}=-\int_{S} \mathbf{S} \cdot \mathbf{n} d S
$$


where the integration goes over the volume $V$ with the surface boundary $S, \mathbf{n}$ is a unit vector in the outward direction normal to the surface, $\mathbf{j}$ is the current density, $\mathbf{S}$ is the Poynting vector, $\mathbf{S}=(c / 4 \pi) \mathbf{E} \times \mathbf{H}$, and $\mathbf{H}$ is the magnetic field. Integrating this equation over time, from $t=-\infty$ to $t=\infty$, and taking into account that at $t= \pm \infty$ there is no electromagnetic field inside the volume $V$, we find

$$
\int_{-\infty}^{\infty} d t \int_{V} d V \mathbf{j} \cdot \mathbf{E}=-\int_{-\infty}^{\infty} d t \int_{S} \mathbf{S} \cdot \mathbf{n} d S
$$

The current density $\mathbf{j}$ includes the current associated with the moving point charge, $\mathbf{j}=$ $e \mathbf{v} \delta(\mathbf{r}(t))$, where $\mathbf{r}(t)$ is the particle's orbit, and the current in the metal. The latter, however, does not contribute to the integral $\int d V \mathbf{j} \cdot \mathbf{E}$, because this integral is equal to the energy deposited inside the metal due to the ohmic heating, which is neglected in the limit of perfect conductivity. Hence the integral reduces to $e \int_{-\infty}^{\infty} \mathbf{v} \cdot \mathbf{E} d t$ taken along the orbit. It is equal to the energy gain (or loss, if negative) $\Delta U$ of the particle due to the interaction with the field. Hence

$$
\Delta U=-\int_{-\infty}^{\infty} d t \int \mathbf{S} \cdot \mathbf{n} d S
$$

Note that this formula is exact, it is valid for arbitrary curvilinear motion of the particle under the influence of an external field.

In calculation of fields, it is convenient to use the Fourier transform, which we define as

$$
\left\{\begin{array}{c}
\mathcal{E}(\omega) \\
\mathcal{H}(\omega)
\end{array}\right\}=\frac{1}{2 \pi} \int_{-\infty}^{\infty} d t e^{i \omega t}\left\{\begin{array}{c}
\mathbf{E}(t) \\
\mathbf{H}(t)
\end{array}\right\} .
$$

Using the Parseval's theorem we find

$$
\begin{aligned}
\Delta U(\mathcal{E}, \mathcal{H}) & =-\frac{c}{2} \int_{-\infty}^{\infty} d \omega \int \operatorname{Re}\left[\mathcal{E}(\omega) \times \mathcal{H}^{*}(\omega)\right] \cdot \mathbf{n} d S \\
& =-\frac{c}{2} \int_{-\infty}^{\infty} d \omega \int \operatorname{Re}\left(\mathcal{E}(\omega) \cdot \mathcal{E}^{*}(\omega)\right) d S
\end{aligned}
$$

where the asterisk denotes complex conjugate, and we used the relations $\mathcal{H}=\mathbf{n} \times \mathcal{E}$ and $\mathbf{n} \cdot \mathcal{E}=0$ valid in the far zone. Below we will use the notation $\Delta U(\mathcal{E}, \mathcal{E})$ for the last integral in Eq. (4) to indicate both fields involved in the calculation of $\Delta U$.

The field entering Eq. (4) is a superposition of the laser field, $\mathcal{E}^{\mathrm{LS}}$, and the particle's field, $\mathcal{E}^{\mathrm{PS}}$ :

$$
\mathcal{E}=\mathcal{E}^{\mathrm{LS}}+\mathcal{E}^{\mathrm{PS}}
$$


where the letter "S" in the subscript indicates that these are the fields in the presence of the screen. For what follows, we will also need a notation the laser field without the screen $\mathcal{E}^{\mathrm{L}}$, and the beam field without the screen, $\mathcal{E}^{\mathrm{P}}$. We define the radiation fields $\mathcal{E}^{\mathrm{LR}}$ and $\mathcal{E}^{\mathrm{PR}}$ as a difference between the field with the screen and the field in free space: $\mathcal{E}^{\mathrm{LR}}=\mathcal{E}^{\mathrm{LS}}-\mathcal{E}^{\mathrm{L}}$, $\mathcal{E}^{\mathrm{PR}}=\mathcal{E}^{\mathrm{PS}}-\mathcal{E}^{\mathrm{P}}$. The radiation fields are generated by currents flowing in the screen. The fields $\mathcal{E}^{\mathrm{LS}}$ and $\mathcal{E}^{\mathrm{PS}}$ can be considered as a superposition of fields without the screen and the radiation fields,

$$
\mathcal{E}^{\mathrm{LS}}=\mathcal{E}^{\mathrm{L}}+\mathcal{E}^{\mathrm{LR}}, \quad \mathcal{E}^{\mathrm{PS}}=\mathcal{E}^{\mathrm{P}}+\mathcal{E}^{\mathrm{PR}}
$$

Substituting Eq. (5) into Eq. (4) we obtain several terms. The term $\Delta U\left(\mathcal{E}^{\mathrm{LS}}, \mathcal{E}^{\mathrm{LS}}\right)$ corresponds to the integrated energy flow of the laser light through the surface $S$ without the beam. This term vanishes because we assume that there are no losses in the screen and hence the incoming laser energy is equal to the outcoming one. The term $\Delta U\left(\mathcal{E}^{\mathrm{PS}}, \mathcal{E}^{\mathrm{PS}}\right)$ describes the energy radiated by the particle passing through the hole in the screen when there is no laser field. This term scales as a square of the particle's charge and is not relevant to the acceleration. Only the cross term

$$
-c \int_{-\infty}^{\infty} d \omega \int \operatorname{Re}\left(\mathcal{E}^{\mathrm{LS}} \cdot \mathcal{E}^{\mathrm{PS} *}\right) d S,
$$

is responsible for the acceleration of the particle.

For calculation, it is convenient to cast Eq. (7) into a different form. Using second equation in Eq. (6) we will represent Eq. (7) as a sum of two terms. The first one involves the particle's field without the screen:

$$
-c \int_{-\infty}^{\infty} d \omega \int \operatorname{Re}\left(\mathcal{E}^{\mathrm{LS}} \cdot \mathcal{E}^{\mathrm{P}^{*}}\right) d S
$$

This term describes interference of the charge's Coulomb field in vacuum with the laser field. In the limit $R \rightarrow \infty$, this term vanishes because the Coulomb field moves with the charge with velocity $v<c$, and the laser light propagates with the speed of light $c$. Since we assume that the laser pulse overlaps with the particle in the vicinity of the hole, at large distance from the hole these two fields will be separated in space. Hence, the particle's acceleration is given by the second term:

$$
\Delta U_{\mathrm{acc}}=-c \int_{-\infty}^{\infty} d \omega \int \operatorname{Re}\left(\mathcal{E}^{\mathrm{LS}} \cdot \mathcal{E}^{\mathrm{PR}^{*}}\right) d S,
$$


for which we use the notation $\Delta U_{\text {acc }}$. Notice that the presence of the field $\mathcal{E}^{\mathrm{PR}}$ in this equation indicates that a particle can only be accelerated if it radiates.

Although in the above derivation we referred to the layout of the acceleration experiment outlined in Fig. 1, our result is not limited by this specific arrangement. With a slight modification, it can also be used for calculation of the energy gain for other accelerator schemes, such as, e.g., inverse FEL or inverse Cerenkov acceleration.

In our calculations of the radiation field below we will assume that the particle moves with a constant velocity. Hence, we will neglect the effect of the laser field on the particle's orbit, as well as the effect of radiation reaction. Such an approximation describes linear acceleration proportional to the laser electric field.

\section{DIFFRACTION RADIATION ON A ROUND HOLE}

Following the approach developed in the previous section, we will first calculate the radiation field $\mathcal{E}^{\mathrm{PR}}$ of the particle. We now assume that the hole in the screen is round, with radius $a$, and consider a relativistic particle moving along the axis of the screen with a constant velocity $v$ close to the speed of light. In the limit of large Lorentz factor, $\gamma \gg 1$, the radial electric and azimuthal magnetic fields of the particle are:

$$
E_{r}^{\mathrm{P}}(r, z, t)=H_{\theta}^{\mathrm{P}}(r, z, t)=\frac{e \gamma r}{\left[r^{2}+\gamma^{2}(z-v t)^{2}\right]^{3 / 2}}
$$

To calculate the radiation field in the far zone we will use diffraction formulae [7-9]. This approach is valid, if the reduces wavelength of the radiation $\lambda=\lambda / 2 \pi$ is much smaller than the radius of the hole $a$, and the diffraction angle is small, $\theta \ll 1$. According to the diffraction theory [7], the field behind the screen $\mathcal{E}^{\mathrm{PS}}$, at large distance $R \rightarrow \infty$ and $z>0$, can be calculated by integration of the incident field $\mathcal{E}^{\mathrm{P}}$ on the screen at $z=0$ :

$$
\mathcal{E}^{\mathrm{PS}}=\frac{e^{i k R}}{R} \frac{i}{2 \pi} \mathbf{k} \times \int_{\text {aperture }} e^{-i \mathbf{k r}} \mathbf{n} \times \mathcal{E}^{\mathrm{P}} d S,
$$

where $\mathbf{r}=(x, y)$ is the two-dimensional vector in the plane of the hole, $\mathbf{k}$ is the wavenumber vector in the direction of the radiation, and $\mathbf{n}$ is the unit vector perpendicular to the surface of the hole. The integration in Eq. (10) goes over the cross section of the hole.

Equation (10) is derived in [7] for the case when the incident wave propagates in free space. In our problem the incident field is the Coulomb field carried by the particle. In this 
case, Eq. (10) gives the total field behind the screen including the field of the particle, and to find the radiation field, we need to subtract the Coulomb field of the electron. The latter can be calculated as the same integral Eq. (10) in the limit $a \rightarrow \infty$, that is when the screen is removed. The result of such a subtraction will be an integral, with the sign opposite to that in Eq. (10), in which the integration goes over the screen surface, rather than the hole [8]:

$$
\mathcal{E}^{\mathrm{PR}}=\mathcal{E}^{\mathrm{PS}}-\mathcal{E}^{\mathrm{P}}=-\frac{e^{i k R}}{R} \frac{i}{2 \pi} \mathbf{k} \times \int_{\text {screen }} e^{-i \mathbf{k r}} \mathbf{n} \times \mathcal{E}^{\mathrm{P}} d S .
$$

A more rigorous proof of this equation can be found in Ref. [9].

The particle's field on the screen is given by $E_{r}(r, 0, t)$ and $H_{\theta}(r, 0, t)$ in Eq. (9). Fourier transformation of these fields defined by Eq. (3) gives:

$$
\mathcal{E}_{r}^{\mathrm{P}}(r, \omega)=\mathcal{H}_{\theta}^{\mathrm{P}}(r, \omega) \approx \frac{k e}{\pi c \gamma} K_{1}\left(\frac{k r}{\gamma}\right)
$$

where $k=\omega / c, K_{1}$ is the modified Bessel function, and we have used $v \approx c$ in the above expression.

In the limit of large $\gamma$, the angle of the radiation relative to the $z$ axis, $\theta$, is small, $\theta \ll 1$. Substituting Eq. (12) into Eq. (11) and neglecting higher-order terms in $\theta$, we find that $\mathcal{E}^{\mathrm{PR}}$ has the radial component only,

$$
\begin{aligned}
\mathcal{E}_{r}^{\mathrm{PR}} & =-k \frac{e^{i k R}}{R} \int_{a}^{\infty} r d r \mathcal{E}_{r}(r, \omega) J_{1}(k r \theta) \\
& =-\frac{e k^{2}}{\pi \gamma c} \frac{e^{i k R}}{R} \int_{a}^{\infty} r d r K_{1}\left(\frac{k r}{\gamma}\right) J_{1}(k r \theta),
\end{aligned}
$$

The integration in the last formula can be carried out analytically [10],

$$
\mathcal{E}_{r}^{\mathrm{PR}}=A(\omega, \theta) \frac{e^{i k R}}{R}
$$

with

$$
A(\omega, \theta)=\frac{e}{\pi \gamma c} \frac{k a}{\theta^{2}+\gamma^{-2}}\left[\theta J_{2}(k a \theta) K_{1}\left(\frac{k a}{\gamma}\right)-\frac{1}{\gamma} J_{1}(k a \theta) K_{2}\left(\frac{k a}{\gamma}\right)\right] .
$$

This formula agrees with the rigorous solution of the diffraction radiation problem obtained in Ref. [11], if one takes the limit $\gamma \gg 1, k a \gg 1$ of their result. In the limit $\lambda \gg a \gamma^{-1}$ (but $\lambda$ still much less than $a$ ) we have [8],

$$
A(\omega, \theta)=-\frac{e}{\pi c} \frac{\theta}{\theta^{2}+\gamma^{-2}} J_{0}(k a \theta)
$$


which in a small-angle approximation yields:

$$
A(\omega, \theta)=-\frac{e}{\pi c} \frac{\theta}{\theta^{2}+\gamma^{-2}}
$$

Since the hole radius $a$ drops out from the last equation, it is also valid in the limit $a \rightarrow 0$, when there is no hole in the screen. In this limit, it is usually called the transition radiation.

Using Eq. (4) it is easy to show that the total radiated energy is given by the integral $c \int_{4 \pi} d \Omega \int_{0}^{\infty} d \omega A^{2}(\omega, \theta)$, where the first integral goes over the solid angle $\Omega=4 \pi$. Hence the spectral density of the diffraction radiation $d^{2} U / d \omega d \theta$ per unit angle $\theta$ is

$$
\frac{d^{2} U}{d \omega d \theta}=2 \pi \theta c A^{2}
$$

Integration over the angle $\theta$ gives the spectrum of the radiation $d U / d \omega$ :

$$
\frac{d U}{d \omega}=2 \int_{0}^{\infty} d \theta \frac{d^{2} U}{d \omega d \theta}=\frac{2}{\pi} \frac{e^{2}}{c} F\left(\frac{a k}{\gamma}\right),
$$

where

$$
F(x)=x^{2}\left[K_{0}(x) K_{2}(x)-K_{1}(x)^{2}\right]
$$

with $K_{n}$ - the modified Bessel functions of the second kind. The factor of 2 in the integral in Eq. (17) takes into account that radiation is symmetric in the forward and backward directions. The function $F$ has a logarithmic singularity at $x=0$.

To find the total radiated energy, we integrate the spectrum over the frequency

$$
U_{\mathrm{rad}}=\int_{0}^{\infty} \frac{d P}{d \omega} d \omega=\frac{3 \pi}{8} \frac{e^{2} \gamma}{a}
$$

It is interesting to note that $U_{\text {rad }}$ is equal to twice the electromagnetic energy of a moving charge in the region $r>a$.

\section{ACCELERATION BY A HIGHER-ORDER LASER MODE}

For the laser field, as in Ref. [3], we first consider a radially polarized TEM $_{10}$ mode with the transverse field:

$$
\mathbf{E}_{\perp}^{\mathrm{L}}(\mathbf{r}, z, t)=E_{0} e^{i k_{\mathrm{L}} z-i \omega_{\mathrm{L}} t} \frac{w_{0}}{w} \frac{\mathbf{r}}{w} \exp \left(-\frac{r^{2}}{w^{2}}-i \frac{k_{\mathrm{L}} r^{2}}{2 f}+2 i \psi\right),
$$


where $\omega_{\mathrm{L}}$ is the laser frequency, $k_{\mathrm{L}}=\omega_{\mathrm{L}} / c=2 \pi / \lambda_{\mathrm{L}}$, the laser waist with a size $w_{0}$ is assumed to be located at the screen, hence

$$
\begin{gathered}
w^{2}=w_{0}^{2}\left(1+\frac{z^{2}}{z_{R}^{2}}\right), \quad z_{R}=\frac{k_{\mathrm{L}} w_{0}^{2}}{2}, \\
f=z+\frac{z_{R}^{2}}{z}, \quad \psi=\arctan \left(\frac{z}{z_{R}}\right) .
\end{gathered}
$$

The choice of this higher-order mode is motivated in part by the fact that it matches the radial polarization of the diffraction radiation in Eq. (14) and is expected to produce better acceleration for the same laser energy.

Equation (10) (with the superscript "P" substituted for "L") enables us to calculate the diffraction of the laser field through the round hole. First, we Fourier transform Eq. (19):

$$
\begin{aligned}
\mathcal{E}^{\mathrm{L}}(\mathbf{r}, z, \omega) & =\frac{1}{2 \pi} \int_{-\infty}^{\infty} d t e^{i \omega t} E_{r}^{\mathrm{L}}(r, z, t) \\
& =E_{0} \delta\left(\omega-\omega_{\mathrm{L}}\right) e^{i k z} \frac{w_{0}}{w} \frac{\mathbf{r}}{w} \exp \left(-\frac{r^{2}}{w^{2}}-i \frac{k r^{2}}{2 f}+2 i \psi\right) .
\end{aligned}
$$

Putting this expression into Eq. (10) yields

$$
\mathcal{E}_{r}^{\mathrm{LS}}(z>0)=E_{0} \delta\left(\omega-\omega_{\mathrm{L}}\right) \frac{e^{i k R}}{R} \frac{(-k)}{w_{0}} \int_{0}^{a} d r r^{2} J_{1}(k r \theta) e^{-r^{2} / w_{0}^{2}},
$$

where, as was defined in Section II, the superscript "LS" stands for the diffracted laser field. Note that for $a \rightarrow \infty$, Eq. (22) can be integrated to yield:

$$
\mathcal{E}_{r}^{\mathrm{LS}}(z>0, a \rightarrow \infty)=-E_{0} \delta\left(\omega-\omega_{\mathrm{L}}\right) \frac{e^{i k z}}{z} \frac{k^{2} w_{0}^{3} \theta}{4} \exp \left(-\frac{k^{2} w_{0}^{2} \theta^{2}}{4}\right)
$$

which is consistent with Eq. (21) in the limit $z \rightarrow \infty$.

As was pointed out in Section II, it is convenient to represent the diffracted laser field as a sum of the original laser field (when the screen is absent), and the field $\mathcal{E}^{\mathrm{LR}}$ due to the radiation of the currents in the screen, $\mathcal{E}^{\mathrm{LS}}=\mathcal{E}^{\mathrm{L}}+\mathcal{E}^{\mathrm{LR}}$. For the radiation field, we have

$$
\mathcal{E}_{r}^{\mathrm{LR}}=E_{0} \delta\left(\omega-\omega_{\mathrm{L}}\right) \frac{e^{i k R}}{R} \frac{k}{w_{0}} \int_{a}^{\infty} d r r^{2} e^{-r^{2} / w_{0}^{2}} J_{1}(k r \theta) .
$$

This field was calculated in the region $z>0$. However, due to the symmetry of the screen, it is symmetric about the point $z=0$. In the region $z<0, \theta$ is then taken to be the angle relative to the $(-z)$ axis; here $\mathcal{E}_{r}^{\mathrm{LR}}$ represents the reflected waves propagating in the direction opposite to the incident laser beam. 
We now calculate the acceleration of this laser mode using the energy balance Eq. (8). Ignoring a pure phase factor, and noting that both fields $\mathcal{E}^{\mathrm{LS}}$ and $\mathcal{E}^{\mathrm{PR}}$ have radial polarization, the energy gain of the particle is

$$
\Delta U_{\mathrm{acc}}=\left|\frac{c}{2} \int_{-\infty}^{\infty} d \omega \int_{S} d S\left[\mathcal{E}_{r}^{\mathrm{LS}}\left(\mathcal{E}_{r}^{\mathrm{PR}}\right)^{*}+\left(\mathcal{E}_{r}^{\mathrm{LS}}\right)^{*} \mathcal{E}_{r}^{\mathrm{PR}}\right]\right|
$$

In the region where $z<0$, only the reflected field $\mathcal{E}^{\mathrm{LR}}$ can interfere with the radiation field since they propagate in the same direction (to the left of the screen in Fig. 1). Writing $\int_{S} d S=R^{2} \int d \Omega=2 \pi R^{2} \int_{0}^{\infty} \theta d \theta$ and inserting Eqs. (22) and (24), we have

$$
\begin{aligned}
\Delta U_{\mathrm{acc}}=\frac{2 \pi c E_{0} k_{\mathrm{L}}}{w_{0}} \mid & -\int_{0}^{\infty} \theta d \theta \frac{e k_{\mathrm{L}}^{2}}{\pi \gamma c} \int_{a}^{\infty} r d r K_{1}\left(\frac{k_{\mathrm{L}} r}{\gamma}\right) J_{1}\left(k_{\mathrm{L}} r \theta\right) \int_{0}^{a} d r^{\prime}\left(r^{\prime}\right)^{2} e^{-\left(r^{\prime}\right)^{2} / w_{0}^{2}} J_{1}\left(k_{\mathrm{L}} r^{\prime} \theta\right) \\
& +\int_{0}^{\infty} \theta d \theta \frac{e k_{\mathrm{L}}^{2}}{\pi \gamma c} \int_{a}^{\infty} r d r K_{1}\left(\frac{k_{\mathrm{L}} r}{\gamma}\right) J_{1}\left(k_{\mathrm{L}} r \theta\right) \int_{a}^{\infty} d r^{\prime}\left(r^{\prime}\right)^{2} e^{-\left(r^{\prime}\right)^{2} / w_{0}^{2}} J_{1}\left(k_{\mathrm{L}} r^{\prime} \theta\right) \mid
\end{aligned}
$$

Using the orthogonality of Bessel functions

$$
\int_{0}^{\infty} \theta d \theta J_{\nu}(k r \theta) J_{\nu}\left(k r^{\prime} \theta\right)=\frac{\delta\left(k r-k r^{\prime}\right)}{k r},
$$

we obtain

$$
\Delta U_{\mathrm{acc}}=\frac{2 e E_{0} k_{\mathrm{L}}}{\gamma w_{0}} \int_{a}^{\infty} r^{2} d r e^{-r^{2} / w_{0}^{2}} K_{1}\left(\frac{k_{\mathrm{L}} r}{\gamma}\right)
$$

Let us first assume that $k_{\mathrm{L}} \ll \gamma / w_{0}$ and use the approximation $K_{1}(x) \approx 1 / x$. Introducing the laser diffraction angle $\alpha_{d}=2 /\left(k_{\mathrm{L}} w_{0}\right)$ the condition for the approximation can be also written as $\alpha_{d} \gg \gamma^{-1}$. Equation (28) then yields

$$
\Delta U_{\mathrm{acc}} \approx e E_{0} w_{0} e^{-a^{2} / w_{0}^{2}}=4 e \sqrt{\frac{2 P_{\mathrm{L}}}{c}} \exp \left(-\frac{a^{2}}{w_{0}^{2}}\right),
$$

where the average power carried by this mode is

$$
\begin{aligned}
P_{\mathrm{L}} & =\frac{c}{8 \pi} \int_{z=0 \text { plane }} d S E_{r}^{\mathrm{L}} H_{r}^{\mathrm{L}} \\
& =\frac{c}{8 \pi} E_{0}^{2} 2 \pi \int_{0}^{\infty} r d r \frac{r^{2}}{w_{0}^{2}} \exp \left(-\frac{2 r^{2}}{w_{0}^{2}}\right)=\frac{c}{32} E_{0}^{2} w_{0}^{2} .
\end{aligned}
$$

Equation (29) shows an important result: in order to accelerate a particle the laser beam should also irradiate the material wall of the screen. If the focal size of the laser light is so small that it does not touch the metal, $w_{0} \ll a$, the acceleration diminishes exponentially. 
For optimal acceleration, we should have $a<w_{0}$ with the maximum energy gain in units of $m c^{2}$ :

$$
\Delta \gamma_{\max }=4 \sqrt{2} \sqrt{\frac{P_{L}}{P_{0}}},
$$

where $P_{0}=m^{2} c^{5} / e^{2} \approx 8.7 \mathrm{GW}$. For a $1 \mathrm{TW}$ laser, we find $\Delta \gamma_{\max } \approx 60$.

In a general case of arbitrary relation between $k_{\mathrm{L}}$ and $\gamma / w_{0}$, Eq. (28) can be rewritten as

$$
\Delta \gamma_{\text {acc }}=4 \sqrt{2} \sqrt{\frac{P_{L}}{P_{0}}} G(A, B),
$$

where

$$
\begin{aligned}
& G(A, B)=2 B \int_{A}^{\infty} d x x^{2} e^{-x^{2}} K_{1}(B x), \\
& A=\frac{a}{w_{0}}, \quad B=\frac{k_{\mathrm{L}} w_{0}}{\gamma}=\frac{2}{\gamma \alpha_{d}} .
\end{aligned}
$$

The maximum of $G$ is 1 when $A=B=0$. When $B=0, G(A, 0)=e^{-A^{2}}$-this is the approximation used in Eq. (29). When $a=A=0$,

$$
G(0, B)=\left[1-\frac{B^{2}}{4} \exp \left(\frac{B^{2}}{4}\right) \Gamma\left(0, \frac{B^{2}}{4}\right)\right],
$$

where $\Gamma(0, Z)=\int_{Z}^{\infty} d t e^{-t} / t$ is the incomplete Gamma function. As shown in Appendix A, Eq. (34) agrees with direct integration of Eq. (1) in the absence of a hole, confirming the validity of this approach.

\section{ACCELERATION BY TWO-CROSSED GAUSSIAN LASER BEAMS}

Another laser acceleration scheme employs a pair of linearly polarized laser beams with the Gaussian fundamental mode focused to the screen and crossed at a small angle to the $z$ axis. If the two identical lasers are out of phase by $\pi$, the transverse components cancel while the longitudinal components add. In the absence of a beam-passage aperture, the acceleration has been directly calculated by integrating the longitudinal field along the beam trajectory [3]. Here we calculate the energy gain from the energy balance Eq. (8). It is sufficient to consider one tilted laser since the total energy gain of two-crossed laser beams is simply twice as large.

First, we calculate the laser field in presence of the screen, following closely the derivation of Section IV. The Gaussian fundamental mode for a small tilt angle $\alpha \ll 1$ at the screen 
location $z=0$ is

$$
\mathcal{E}_{x}^{\mathrm{L}}(\mathbf{r}, z=0, \omega) \approx E_{0} \delta\left(\omega-\omega_{\mathrm{L}}\right) e^{i k x \sin \alpha} \exp \left(-\frac{r^{2}}{w_{0}^{2}}\right) .
$$

The diffraction integral can be evaluated as [7]

$$
\mathcal{E}_{x}^{\mathrm{LS}}(z>0)=E_{0} \delta\left(\omega-\omega_{\mathrm{L}}\right) \frac{-i k e^{i k R}}{R} \int_{0}^{a} r d r \exp \left(-\frac{r^{2}}{w_{0}^{2}}\right) J_{0}(k r \xi),
$$

where $\xi=\left(\theta^{2}+\alpha^{2}-2 \theta \alpha \cos \phi\right)^{1 / 2}$, and $\phi$ is the azimuthal angle of the wave vector $\mathbf{k}$ with respect to the $z$ axis. In the region where $z<0$, the total laser field is the incident field and the reflected field given by

$$
\mathcal{E}_{x}^{\mathrm{LR}}=E_{0} \delta\left(\omega-\omega_{\mathrm{L}}\right) \frac{i k e^{i k R}}{R} \int_{a}^{\infty} r d r \exp \left(-\frac{r^{2}}{w_{0}^{2}}\right) J_{0}(k r \xi) .
$$

To compute Eq. (8), we note that $\left|\mathcal{E}^{\mathrm{LS}} \cdot \mathcal{E}^{\mathrm{BS} *}\right|=\left|\mathcal{E}_{x}^{\mathrm{LS}} \mathcal{E}_{r}^{\mathrm{BR}} \cos \phi\right|$ and make use of the Bessel function expansion [10]

$$
J_{0}(k r \xi)=\sum_{m=-\infty}^{\infty} J_{m}(k r \theta) J_{m}(k r \alpha) e^{i m \phi}
$$

Integration over $\phi$ picks up only $m= \pm 1$ terms. Then following the integration steps of Section IV, we find

$$
\begin{aligned}
\Delta U_{\mathrm{acc}} & =2 \frac{2 e E_{0} k_{\mathrm{L}}}{\gamma} \int_{a}^{\infty} r d r K_{1}\left(\frac{k_{\mathrm{L}} r}{\gamma}\right) \exp \left(-\frac{r^{2}}{w_{0}^{2}}\right) J_{1}\left(k_{\mathrm{L}} r \alpha\right) \\
& \approx 4 e E_{0} w_{0} \int_{a / w_{0}}^{\infty} d x e^{-x^{2}} J_{1}\left(\frac{2 \alpha}{\alpha_{d}} x\right)
\end{aligned}
$$

where the extra factor of 2 on the right hand side takes into account two-crossed laser beams, and we have assumed that $k_{\mathrm{L}} \ll \gamma / a$ to use $K_{1}(x) \approx 1 / x$ for the approximate expression. For a vanishing hole as $a \rightarrow 0$, we have

$$
\Delta U_{\mathrm{acc}}=2 e E_{0} w_{0} \frac{\alpha_{d}}{\alpha}\left[1-\exp \left(-\frac{\alpha^{2}}{\alpha_{d}^{2}}\right)\right],
$$

in agreement with Ref. [3] when the injection point is at $z_{I}=-\infty$ and the extraction point is at $z_{F}=0$. At the optimal tilt angle $\alpha_{\text {opt }} \approx 1.1 \alpha_{d}$, the maximum energy gain is $1.3 e E_{0} w_{0}$. For an arbitrary a, Eq. (39) can be used to obtain the optimal tilt angle and the maximum energy gain (see Figs. 2 and 3). As shown in Fig. 3, the maximum energy gain in units of $m c^{2}$ can be approximated by

$$
\Delta \gamma_{\max } \approx 3.6 \sqrt{\frac{P_{\mathrm{L}}}{P_{0}}} \exp \left(-\frac{a^{2}}{w_{0}^{2}}\right) .
$$


Here $P_{\mathrm{L}}=c E_{0}^{2} w_{0}^{2} / 8$ is the total laser power for the two-crossed Gaussian beams. Comparing with Eq. (29), the energy gain of the two-crossed Gaussian laser beams has essentially the same exponential dependence on the radius of the hole. For the same laser power, the radially polarized $\mathrm{TEM}_{10}$ mode is more effective for acceleration (by about a factor of 1.6) because it matches the polarization of the diffraction radiation in this accelerating structure (see Section VIB for more discussions).

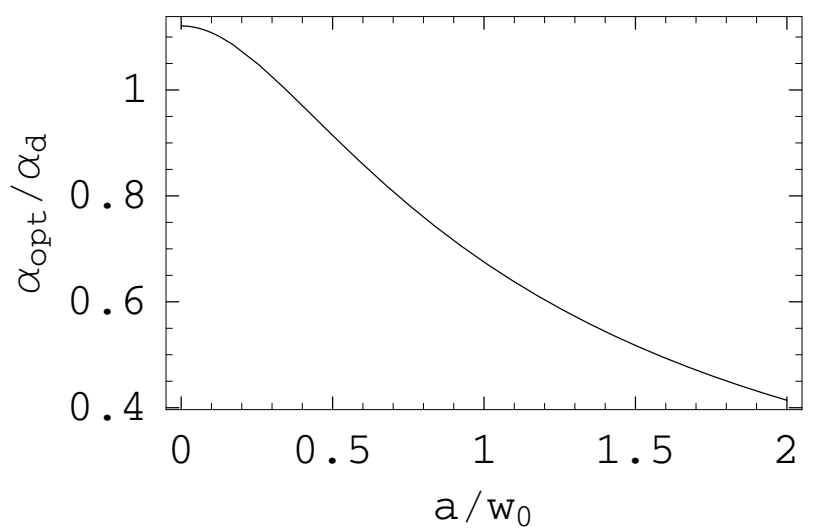

FIG. 2: The optimal tilt angle as a function of the hole radius for the tilted Gaussian laser beam.

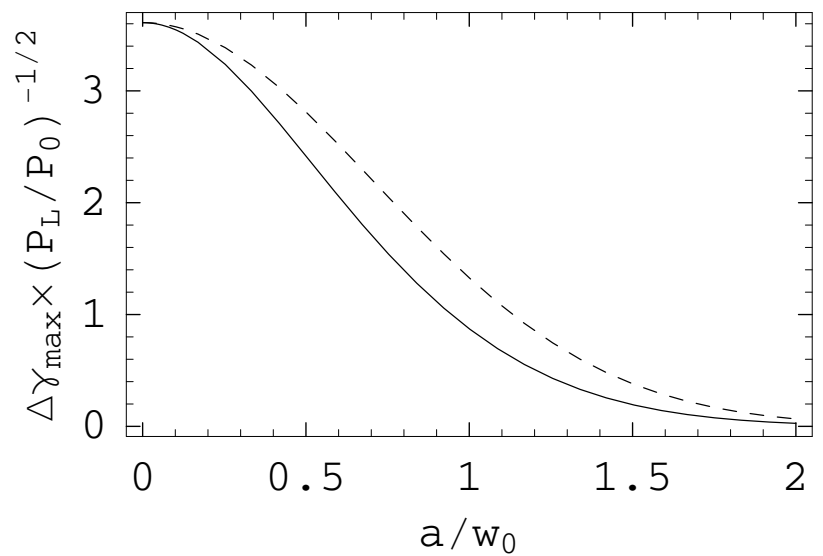

FIG. 3: The maximum energy gain of two-crossed laser beams evaluated at the optimal tilt angle from Eq. (39) (solid line), and compared with the approximate Eq. (41) (dashed line). 


\section{DISCUSSION}

\section{A. Limitation due to material damage}

Results of previous sections suggest that the laser should irradiate the acceleration structure, which is subject to material damage at a certain threshold laser fluence. Considering the case of the higher-order laser mode in Section IV, we rewrite Eq. (32) as

$$
\Delta \gamma_{\mathrm{acc}}=4 \sqrt{2}\left(\frac{U_{\mathrm{L}}}{w_{0}^{2} P_{0} t_{\mathrm{L}}}\right)^{1 / 2} w_{0} G(A, B) \approx\left(\frac{F_{\mathrm{L}}}{P_{0} t_{\mathrm{L}}}\right)^{1 / 2} \gamma \lambda_{\mathrm{L}} B G(A, B),
$$

where $U_{\mathrm{L}}$ is the laser flash energy, $t_{\mathrm{L}}$ is the laser pulse duration, and $F_{\mathrm{L}} \approx U_{\mathrm{L}} / w_{0}^{2}$ is the laser fluence. Since the laser fluence at the material damage threshold is known to be $F_{\text {th }} \approx$ $2 \mathrm{~J} / \mathrm{cm}^{2}$ for sub-ps laser pulses [12], Eq. (42) allows us to optimize the laser spot size or the diffraction angle at the damage threshold for a given laser pulse duration. Figure 4 shows the optimal diffraction angle that maximizes $B G(A, B)$ in Eq. (42). We see that $\left(\alpha_{d}\right)_{\mathrm{opt}} \approx 1 / \gamma$ (i.e., $\left.\left(w_{0}\right)_{\mathrm{opt}} \approx \gamma \lambda_{\mathrm{L}} / \pi\right)$ when $A=a / w_{0}<1$. At the optimal $B_{0}=k_{\mathrm{L}}\left(w_{0}\right)_{\mathrm{opt}} /(\gamma)$, we have $B_{0} G\left(A, B_{0}\right) \approx 0.8$ for a small $A$ from Fig. 5. If we take a typical short-pulse laser with $t_{\mathrm{L}} \sim 100 \mathrm{fs}$, the maximum fractional energy gain limited by the fluence damage threshold is approximately

$$
\frac{\Delta \gamma_{\max }}{\gamma} \approx 0.8 \lambda_{\mathrm{L}} \sqrt{\frac{F_{\mathrm{th}}}{P_{0} t_{\mathrm{L}}}} \approx 4 \times 10^{-3}
$$

Since the effective interaction distance is on the order of the Rayleigh length, we have $z_{R}=\pi w_{0}^{2} / \lambda_{\mathrm{L}} \approx \gamma^{2} \lambda_{\mathrm{L}} / \pi$ at the optimal spot size. The effective acceleration gradient is

$$
\frac{\Delta U_{\mathrm{acc}}}{z_{R}} \approx \frac{8 \times 10^{-3} \gamma m c^{2}}{\gamma^{2} \lambda_{\mathrm{L}} / \pi} \approx \frac{6}{\gamma} \mathrm{GeV} / \mathrm{m}
$$

For a $50 \mathrm{MeV}$ electron (i.e., $\gamma \approx 100$ ), the energy gain is about $200 \mathrm{keV}$ from Eq. (43), and the acceleration gradient is about $60 \mathrm{MeV} / \mathrm{m}$ according to Eq. (44), in agreement with the expected performance of the E-163 proposal [5]. Finally, for the 100 fs laser pulse considered here, the flash energy required to operate at the damage threshold and at the optimal spot size scales as

$$
U_{\mathrm{L}} \approx F_{\mathrm{th}} w_{0}^{2} \approx 2 \times 10^{-6} \gamma^{2} \mathrm{~mJ}
$$




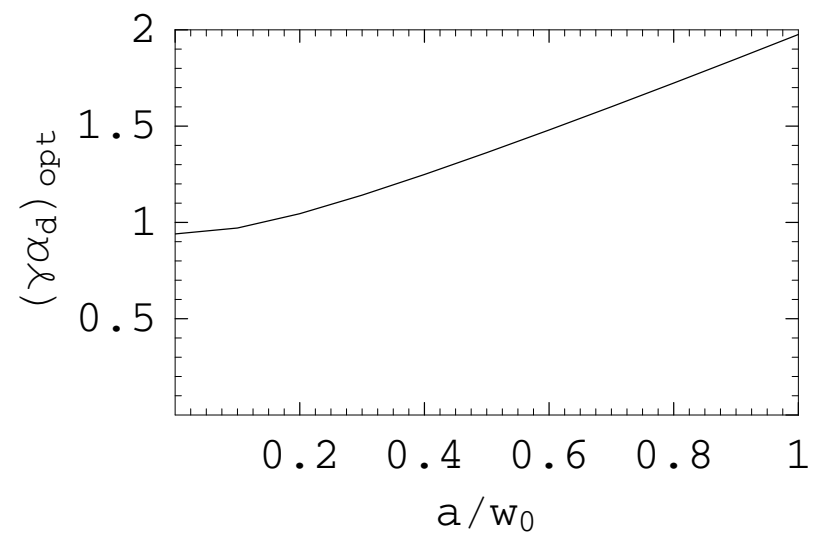

FIG. 4: The optimal laser diffraction angle as a function of the hole radius at the material damage threshold.

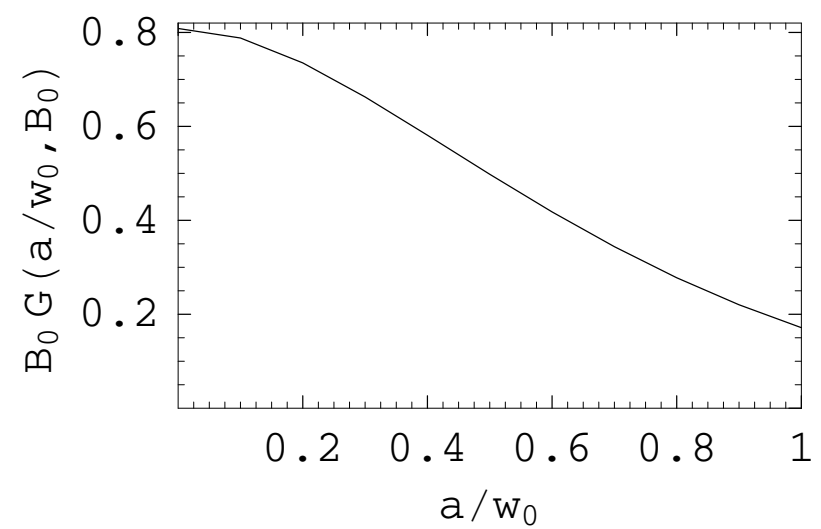

FIG. 5: The function $B_{0} G\left(A, B_{0}\right)$ in Eq. (42) evaluated at the optimal spot size or diffraction angle.

\section{B. Optimal Laser Profile}

As was pointed out in Section $\mathrm{V}$, the radially-polarized $\mathrm{TEM}_{10}$ mode is more effective for laser acceleration than the tilted Gaussian fundamental mode because it matches better with the diffraction radiation pattern. For optimal acceleration, one might consider to shape the laser transverse profile in such a way that achieves maximum acceleration for a given laser power. It is easy to see from calculations in Section IV, that for the optimum acceleration the angular distribution of the reflected laser light $\mathcal{E}^{\mathrm{LR}}$ must match exactly the angular 
distribution of the particle's radiation. In the case $a \ll w_{0}$, this means (see Eq. (16)):

$$
\mathcal{E}_{r}^{\mathrm{LR}}=E_{0} w_{0} \delta\left(\omega-\omega_{\mathrm{L}}\right) \frac{e^{i k z}}{z} \frac{\theta}{\theta^{2}+\gamma^{-2}}
$$

The corresponding laser power $P_{\mathrm{L}} \approx(\ln \gamma) c E_{0}^{2} w_{0}^{2} / 4$ for $\gamma \gg 1$. Integrating the energy balance Eq. (8) then yields:

$$
\Delta \gamma_{\max }=(2 \ln \gamma) e E_{0} w_{0}=4 \sqrt{\ln \gamma} \sqrt{\frac{P_{\mathrm{L}}}{P_{0}}} .
$$

We see that the optimal laser profile (with the angular distribution of the transition ra-

diation) only improves the maximum energy gain by a small factor $\sim \sqrt{\ln \gamma}$ even for an ultra-relativistic particle.

\section{CONCLUSION}

In summary, linear acceleration by a laser field in vacuum is only possible if a particle radiates in passing the accelerating structure. In this paper, we express the energy gain by the particle as an interference integral of the laser field and the radiation field in the far zone and hence avoid calculation of any near field that accelerates the particle. We apply this new method to study laser acceleration in a simple accelerating structure (a conducting screen with a beam-passing hole) and to optimize gain for given laser parameters. We show that for optimal acceleration, the laser should irradiate on the accelerating structure (i.e., the dimension of the hole should be less than the laser spot size), and the laser diffraction angle (as well as the crossing angle in the case of the two-crossed Gaussian beams) should be comparable to the radiation opening angle $\gamma^{-1}$. Limited by the damage threshold fluence, the maximum energy gain in this accelerating structure is proportional to the electron energy, but the acceleration gradient scales as $\gamma^{-1}$.

\section{APPENDIX A: COMPARISON WITH DIRECT CALCULATION OF ACCEL- ERATION}

In the case with no hole, $a=0$, when the screen stops the laser beam from propagating to $z>0$ region the energy gain in a laser field can be calculated directly. Particles will be accelerated by the laser beam in $z<0$ region and stop interacting with the laser beam after 
passing through the screen. (In the absence of the screen, laser would decelerate electrons in $z>0$ region so that the net energy gain is zero.)

For the laser mode given by Eq. (19), the longitudinal electric field can be found from the Maxwell equation $\nabla \cdot \mathbf{E}^{\mathrm{L}}=0$ and is approximated by

$$
\begin{aligned}
E_{z}^{\mathrm{L}} & \approx \frac{i}{k_{\mathrm{L}}} \nabla_{\perp} \cdot \mathbf{E}_{\perp}^{\mathrm{L}}=\frac{i}{k_{\mathrm{L}}} \frac{1}{r} \frac{\partial}{\partial r}\left(r E_{r}^{\mathrm{L}}\right) \\
& =E_{0} e^{i k_{\mathrm{L}} z-i \omega_{\mathrm{L}} t} \frac{w_{0}}{w}\left[\frac{i}{k_{\mathrm{L}}} \frac{2}{w}\left(1-\frac{r^{2}}{w^{2}}\right)+\frac{r^{2}}{w f}\right] \exp \left(-\frac{r^{2}}{w^{2}}-i \frac{k_{\mathrm{L}} r^{2}}{2 f}+2 i \phi\right) .
\end{aligned}
$$

Consider a relativistic particle moving in the $z$-direction along the axis of the system, $\mathbf{r}=0$ and $z=v t$, the energy gain can be obtained by integrating the longitudinal laser field alone the particle trajectory from $z=-\infty$ to $z=0$ (the location of the screen):

$$
\begin{aligned}
\Delta U_{\mathrm{acc}} & =\int_{-\infty}^{0} d z e E_{z}^{\mathrm{L}}(\mathbf{r}=0, t=z / v)=\frac{2 e E_{0}}{k_{\mathrm{L}} w_{0}} \int_{-\infty}^{0} d z \frac{i}{\left(1-i z / Z_{R}\right)^{2}} \exp \left[i k_{\mathrm{L}} z\left(1-\frac{c}{v}\right)\right] \\
& =e E_{0} w_{0}\left[1-\frac{B^{2}}{4} \exp \left(\frac{B^{2}}{4}\right) \Gamma\left(0, \frac{B^{2}}{4}\right)\right]
\end{aligned}
$$

where $B=k_{\mathrm{L}} w_{0} /(\gamma)$, and the square bracket term describes the gain reduction due to relative slippage of the particle to the laser field. This expression is identical to Eq. (34) derived using the energy balance Eq. (8). An approximate expression of Eq. (A2) is given in Ref. [3].

[1] R. B. Palmer, in P. Schoessow, ed., Advanced Accelerator Concepts (American Institute of Physics, New York, 1995), no. 335 in AIP Conference Proceedings, pp. 90-100.

[2] F. Caspers and E. Jensen, Particle Acceleration with the Axial Electric Field in a TEM10 Mode Laser Beam, Tech. Rep. CERN/PS 89-69, CERN (December 1989).

[3] E. Esarey, P. Sprangle, and J. Krall, Phys. Rev. E 52, 5443 (1995).

[4] R. Byer, T. Plettner, C. Barnes, E. Colby, B. Cowan, R. Siemann, and J. Spencer, in Proceedings of the 2001 Particle Accelerator Conference (IEEE, Piscataway, NJ, 2001).

[5] C. Barnes, E. Colby, B. Cowan, R. Noble, D. Palmer, R. Siemann, J. Spencer, and D. Walz, Laser Acceleration of Electrons in Vacuum, Tech. Rep. SLAC Proposal E-163, SLAC (August 2001).

[6] L. D. Landau and E. M. Lifshitz, The Classical Theory of Fields, vol. 2 of Course of Theoretical Physics (Pergamon, London, 1979), 4th ed., (Translated from the Russian). 
[7] J. D. Jackson, Classical Electrodynamics (Wiley, New York, 1975), 2nd ed.

[8] M. Ter-Mikaelian, High-Energy Electromagentic Porcesses in Condensed Media (WileyInterscience, 1972).

[9] B. M. Bolotovskii and E. A. Galst'yan, Physics-Uspekhi 43, 755 (2000).

[10] I. Gradshteyn and I. Ryzhik, Table of integrals, series, and products (Academic Press, 2000), 6 th ed.

[11] G. Dome, E. Gianfelice, L. Palumbo, V. G. Vaccaro, and L. Verolino, Nuovo Cimento A 104, 1241 (1991).

[12] B. Stuart, M. Feit, A. Rubenchik, B. W. Shore, and M. Perry, Phys. Rev. Lett. 74, 2248 (1995). 